\title{
Development of low-cost, root collar diameter measurement devices for pine seedlings
}

\author{
T.E. Grift ${ }^{\mathrm{a}, *}$, R. Oberti ${ }^{\mathrm{b}}$ \\ a Department of Agricultural and Biological Engineering, University of Illinois, 1304 West Pennsylvania Avenue, Urbana, IL 61801, USA \\ ${ }^{\mathrm{b}}$ Institute for Agricultural Engineering, Università degli Studi di Milano, Milano, Italy
}

Received 8 August 2005; received in revised form 1 October 2005; accepted 18 January 2006

\begin{abstract}
To assess the growth stage of pine seedlings in the field, nurseries annually measure the root collar diameter (RCD) of a large number of seedlings. Traditionally this is done manually using a slide micrometer and registration on paper, a tedious and errorprone practice. As an alternative, two generic semi-automatic diameter measurement devices were developed, based on an optical time-of-flight principle. Here the diameter was obtained using the timing information generated by the shadow of an object passing a pair of receivers. In the first method named the 'sliding-edge device', the operator manually slides the object along a guiding edge after which the diameter is measured and recorded automatically. In the second method named the 'counterbalanced device' the operator places the object on a table after which the device measures and records the diameter at the push of a button. Both devices were tested using cylindrical objects with known diameters. The sliding-edge device produced a maximum systematic error of $0.3 \mathrm{~mm}(9.5 \%)$, most likely owing to inaccurate model assumptions and a maximum variability of $0.1 \mathrm{~mm}$. The counterbalanced device proved superior with a maximum systematic error of $0.1 \mathrm{~mm}(1 \%)$, virtually independent of operator skill, and a maximum variability of $0.1 \mathrm{~mm}$.
\end{abstract}

(C) 2006 Elsevier B.V. All rights reserved.

Keywords: RCD; Time-of-flight; Optical; OptoSchmitt

\section{Introduction}

Seedling production in the USA is an industry with 1.6 billion seedlings produced in the year 1997, of which $79 \%$ were in the Southern states (Moulton, 1999). The annual number of seedlings grown per nursery typically varies between 10 and 30 million. The total acreage of tree planting has remained stable during the period 1999-2002, with 2.3 million acres per year (USDA Forest Service, 2002). An overview of tree planting activity in the USA from 1925 to date can be found in South (2005).

An important characteristic of a pine seedling is the root collar diameter (RCD) which is considered the best morphological measurement of seedling quality as reflected in outplanting performance. The RCD is measured in the bare zone between the root and shoot sections and serves to classify seedlings with a resolution of $0.5 \mathrm{~mm}$. Seedling nurseries measure the RCD of thousands of seedlings every year to assess their value in the field. Traditionally this

\footnotetext{
* Corresponding author. Tel.: +1 217333 2854; fax: +1 2172440323.

E-mail address: grift@uiuc.edu (T.E. Grift).
} 


\begin{tabular}{|c|c|}
\hline \multicolumn{2}{|c|}{ Nomenclature } \\
\hline$a$ & receiver's vertical acceleration $\left(\mathrm{m} \mathrm{s}^{-2}\right)$ \\
\hline$A$ & $\begin{array}{l}\text { counterbalanced device constant proportional to the mass imbalance between counterweight and } \\
\text { receivers arm }(\mathrm{kg} \mathrm{m})\end{array}$ \\
\hline$b$ & distance between centers of photo-receivers $(\mathrm{m})$ \\
\hline$b_{\mathrm{H}}$ & distance where object interrupts light beams in center $(\mathrm{m})$ \\
\hline$c$ & counterbalanced device system constant, function of the system's geometry parameters $p$ and $b(\mathrm{~m})$ \\
\hline$D$ & diameter of the measured object $(\mathrm{m})$ \\
\hline$g$ & gravity acceleration $\left(\mathrm{m} \mathrm{s}^{-2}\right)$ \\
\hline$J$ & polar moment of inertia of the counterbalanced device with respect to the pivot point $\left(\mathrm{kg} \mathrm{m}^{2}\right)$ \\
\hline$L$ & distance from light source to receivers $(\mathrm{m})$ \\
\hline$p$ & constant distance from initial position $x_{0}$ to $x\left(t_{1}\right)$ in the counterbalanced configuration (m) \\
\hline$S$ & distance from receivers to sliding edge $(\mathrm{m})$ \\
\hline$\Delta t_{\mathrm{f}}$ & flank separation signal (s) \\
\hline$\Delta t_{\mathrm{p}}$ & pulse width of a single receiver signal (s) \\
\hline$T$ & distance from object path to receivers $(\mathrm{m})$ \\
\hline$v$ & object/receiver velocity $\left(\mathrm{m} \mathrm{s}^{-1}\right)$ \\
\hline$x$ & object/receivers location (m) \\
\hline \multicolumn{2}{|c|}{ Greek letters } \\
\hline$\alpha$ & diverging angle of the light beam (rad) \\
\hline$\theta$ & angle between the horizontal axis and the counterweight-receiver linkage (rad) \\
\hline
\end{tabular}

is done manually using a slide micrometer, a tedious and error prone procedure. Automation of the measurement and administration process would be very helpful considering the volume of labor involved.

Presence detection has been used for seedling counting by several researchers (Maw et al., 1986; Kranzler, 1988; Gasvoda and Herzberg, 1992). With the availability of low-cost cameras and computers, machine vision approaches have been used to determine seedling properties including the RCD (Rigney and Kranzler, 1988; Wilhoit et al., 1994). An overview of the feasibility of machine vision for seedling grading was reported in Kranzler and Rigney (1989). Cameras have also been used to measure standing tree diameters in the field (Clark et al., 2000; Byrne and Singh, 1998). Maw et al. (1986) concluded that the most promising sensor for seedling presence detection was a photointerrupter configuration because of its non-intrusive nature and detection speed. The methods described in this paper are indeed based on photo-interruption, applied in a time-of-flight configuration, where the RCD is obtained from timing information. The same principle was used by McDonald et al. (2003) to develop a real-time diameter sensor for tree length harvesting. Similarly, Delwiche and Voorhees (2003) developed a reflective laser based system to count and measure the diameters of deciduous trees in a field situation. In addition, Grift and Hofstee (1997) used the timeof-flight principle to measure the velocity and diameters of fertilizer particles. The objective of this study was the development and evaluation of lab-scale, time-of-flight based diameter measurement devices, which are inexpensive and operator friendly with an accuracy of $0.1 \mathrm{~mm}$, to accommodate $0.5 \mathrm{~mm}$ RCD classes.

\section{Time-of-flight diameter measurement principle}

The photo-interrupter based time-of-flight measurement principle relies on the shadow casting effect of an object on a pair of receivers. The left side of Fig. 1 shows the interruption process where a cylindrical object with diameter $D$ interrupts two parallel light beams, while moving downward.

Four distinct events in time can be distinguished when the object passes the light beams. At time $t_{1}$ the object reaches the upper light beam and blocks receiver $S_{1}$ and at $t_{2}$, the object reaches the lower light beam and blocks receiver $S_{2}$. Similarly, at time $t_{3}$ the object unblocks receiver $S_{1}$ and at time $t_{4}$ the object unblocks receiver $S_{2}$. The receivers $S_{1}$ and $S_{2}$ are binary optical switches, and their signals during the passage of the object are shown on the right side of Fig. 1. 

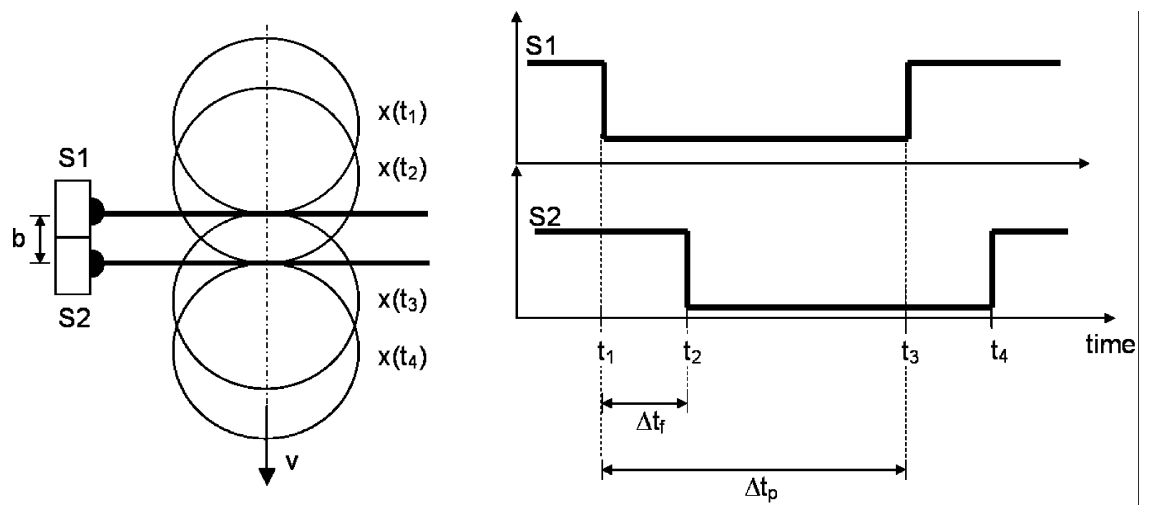

Fig. 1. Relationship between the motion $x(t)$ of an object passing through a dual light beam sensed by a photo-receiver pair $S_{1}$ and $S_{2}$ (left) and their signal output timing (right).

Fig. 1 shows that the distance between the receivers $b$ as well as the object diameter $D$ can be expressed in terms of the corresponding interruption times as follows:

$$
b=x\left(t_{2}\right)-x\left(t_{1}\right)
$$

and

$$
D=x\left(t_{3}\right)-x\left(t_{1}\right)
$$

For convenience, the timing differences were defined as follows (see Fig. 1):

$$
\begin{aligned}
& \Delta t_{\mathrm{f}}=t_{2}-t_{1} \\
& \Delta t_{\mathrm{p}}=t_{3}-t_{1}
\end{aligned}
$$

If the geometry of the system and the equation of motion $x(t)$ are known, Eq. (2) can be used to derive an expression for the object diameter $D$ in terms of the measured times $\Delta t_{\mathrm{p}}, \Delta t_{\mathrm{f}}$ and the geometrical constants of the system (in this case only $b$, the distance between the receivers). Although for cylindrical objects such as shown in Fig. 1, the measured length is equal to the diameter, in essence only a single major axis of the object is measured.

\section{Materials and methods}

Four RCD measurement arrangements were considered, where the seedling: (1) moves with constant velocity interrupting two parallel light beams; (2) moves with constant velocity interrupting a diverging light beam; (3) slides along an edge with constant velocity interrupting a diverging light beam; (4) rests in the path of a counterbalanced, free-falling arm holding the receivers, interrupting two diverging beams.

Case 1 and 2 are either expensive to produce or impractical; they are discussed since they form the bases of case 3 and 4. The sliding-edge and counterbalanced measurement devices were considered candidates for practical use and they were implemented using the following components. The receivers consisted of a pair of infrared ( $940 \mathrm{~nm}$ peak) 'OptoSchmitts' (Honeywell, SDP8601), which contain a photodiode, amplifier, voltage regulator, Schmitt trigger and an NPN output transistor with a $10 \mathrm{kOhm}$ pull-up resistor. The receivers are molded in a side-looking black plastic package to minimize the effect of visible ambient light and the sensitivity of the receiver photodiode is internally temperature compensated.

For the light source, a high-output $940 \mathrm{~nm}$ infrared light emitting diode (RadioShack, 276-143) was used. For reliable operation, this diode must deliver at least $2.5 \mathrm{~mW} / \mathrm{cm}^{2}$ of radiation at the receiver and to ensure consistent switching points, its output has to remain constant. The timing signals $\Delta t_{\mathrm{p}}, \Delta t_{\mathrm{f}}$ were measured with a counter/timer board (National Instruments, PCI-6601), under control of LabView ${ }^{\circledR}$. 


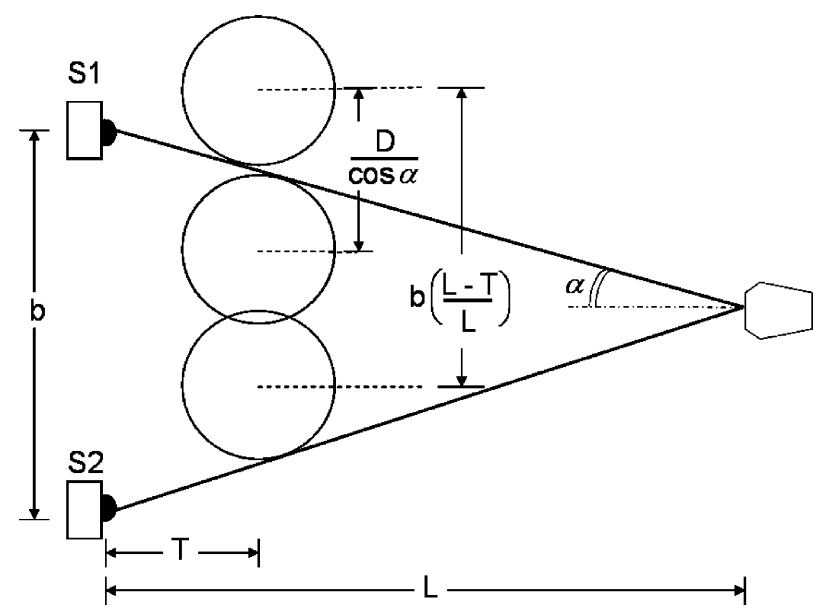

Fig. 2. Measurement configuration with a diverging light beam.

\subsection{Parallel light beam case with constant object velocity}

This configuration assumes two parallel light beams through which the user moves the object. The parallel beam with constant object velocity is very simple in theory, but in practice hard to implement. A truly parallel beam requires collimation of the light source and to ensure a constant velocity and object orientation, extra handling hardware is needed. It is discussed here as an introduction to the more practical concepts. Under the assumption of a constant velocity and parallel light beams the equation of motion $x(t)$ becomes linear (Fig. 1):

$$
x(t)=v_{0} t+x_{0}
$$

where $v_{0}$ is the unknown constant velocity, and $x_{0}$ is the position at an arbitrary initial time $t=0$. Applying Eq. (5) for $t=t_{1}$ and $t=t_{2}$ and using Eqs. (1) and (3), allows the velocity to be written as

$$
v_{0}=\frac{x\left(t_{2}\right)-x\left(t_{1}\right)}{\left(t_{2}-t_{1}\right)}=\frac{b}{\Delta t_{\mathrm{f}}}
$$

Similarly, applying Eq. (5) for $t=t_{1}$ and $t=t_{3}$ and using Eqs. (2) and (4) yields an expression for the unknown diameter in terms of the time differences $\Delta t_{\mathrm{p}}, \Delta t_{\mathrm{f}}$ :

$$
D=v_{0} \Delta t_{\mathrm{p}}=b \frac{\Delta t_{\mathrm{p}}}{\Delta t_{\mathrm{f}}}
$$

Although the velocity is assumed constant during measurement, the diameter measurement is independent of the absolute value of the velocity itself. For instance, if the velocity doubles, $\Delta t_{\mathrm{f}}$ would halve, but since $\Delta t_{\mathrm{p}}$ would halve as well, the ratio remains constant and the diameter measurement unaffected.

\subsection{Diverging light beam case with constant object velocity}

The diverging beam is simpler and less expensive to implement than a parallel beam, since a single transmitter with an appropriate aperture angle can be used in combination with two receivers. The operator must still maintain a constant velocity during measurement, but more importantly she/he must move the object center past the receivers at a constant distance and vertical orientation, which is impractical. It is discussed here since its principle is used in both implemented methods. Fig. 2 shows the light interruption process for an object with diameter $D$, moving downward at a constant velocity $v_{0}$ in the diverging light beam arrangement. Note that the object center is assumed to pass the receivers at a constant distance $(T)$.

Fig. 2 shows that, due to the beam's diverging angle $2 \alpha$, the actual space traveled by the object to block the two receivers with distance $b$ (the space traveled during the flank time $\Delta t_{\mathrm{f}}$ ) is reduced by the ratio $(L-T) / L$, where $L$ represents the distance from the light source to the receiver plane, and $T$ the distance from the object center trajectory 




Fig. 3. Lab-scale, sliding-edge diameter measurement device.

to the receiver plane. Consequently Eq. (6), the expression for the velocity in the parallel beam case, becomes:

$$
\Delta t_{\mathrm{f}}=\frac{b}{v_{0}}\left(\frac{L-T}{L}\right)
$$

Similarly, the actual space traveled by the object while passing one receiver (the space traveled during the pulse time $\left.\Delta t_{\mathrm{p}}\right)$ is larger than $D$ by a factor $(1 / \cos \alpha)$. Thus Eq. (7), the expression for the diameter in the parallel beam case, rewritten as $\Delta t_{\mathrm{p}}=D / v_{0}$ becomes:

$$
\Delta t_{\mathrm{p}}=\left(\frac{D}{\cos \alpha}\right) \frac{1}{v_{0}}
$$

Combining Eqs. (8) and (9) and solving for $D$ gives:

$$
D=b\left(\frac{L-T}{L}\right) \frac{\Delta t_{\mathrm{p}}}{\Delta t_{\mathrm{f}}} \cos \alpha
$$

The geometry in Fig. 2 shows that:

$$
\cos \alpha=\frac{L}{\sqrt{L^{2}+(b / 2)^{2}}}
$$

The expression for the diameter measurement in the diverging beam case becomes now:

$$
D=b \frac{\Delta t_{\mathrm{p}}}{\Delta t_{\mathrm{f}}}\left(\frac{L-T}{\sqrt{L^{2}+(b / 2)^{2}}}\right)
$$

\subsection{Sliding-edge case with constant object velocity}

To make the previous concepts more practical, a first addition was a sliding edge which ensures a constant distance from the edge of the object to the receivers. This idea was implemented as shown in Fig. 3.

The principle of the sliding-edge configuration with diverging beam is shown in Fig. 4.

Whereas in the diverging beam configuration without sliding edge, the distance between the object center and the receivers was constant ( $T$, see Fig. 2), now the distance $S$ between the object edge and the receivers becomes constant. 


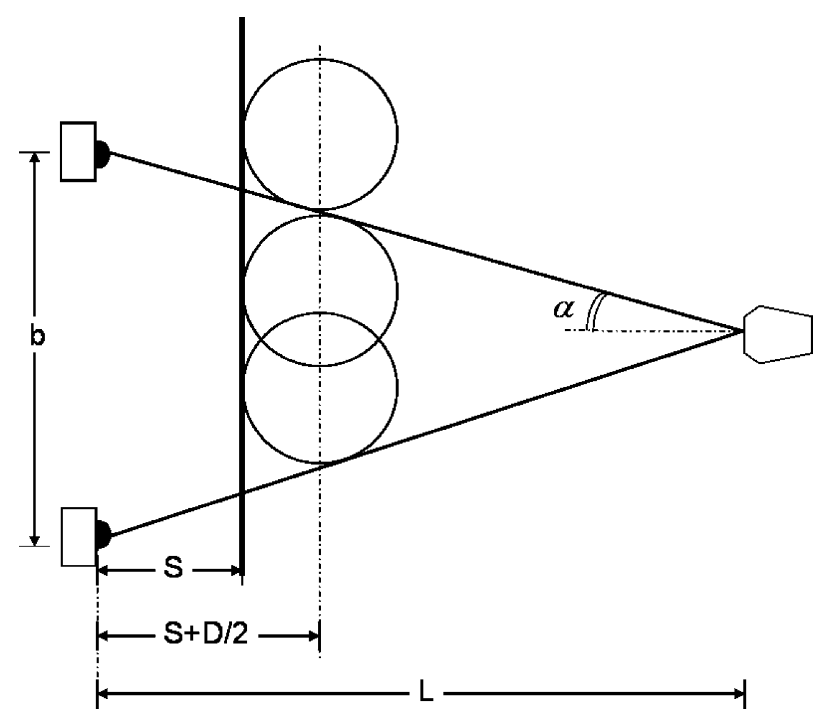

Fig. 4. Measurement configuration with a diverging light beam and an edge of the object sliding along a guide at a distance $S$ from the receivers.

This means that Eq. (12) can be adapted to the sliding-edge configuration by substituting $S+D / 2$ for $T$ leading to

$$
D=b \frac{\Delta t_{\mathrm{p}}}{\Delta t_{\mathrm{f}}}\left(\frac{L-(S+(D / 2))}{\sqrt{L^{2}+(b / 2)^{2}}}\right)
$$

Solving for $D$ gives:

$$
D=\frac{L-S}{\left((1 / 2)+\left(\Delta t_{\mathrm{f}} / \Delta t_{\mathrm{p}}\right) \sqrt{(L / b)^{2}+(1 / 4)}\right)}
$$

\subsection{Counterbalanced configuration with constant receiver acceleration}

The manual sliding system as described earlier was expected to be prone to operator-induced errors because of the requirement to slide the object at a constant speed while maintaining a vertical object orientation. Alternatively, a configuration was developed where the receivers move with respect to the object, rather than vice versa. Fig. 5 shows the receivers in rest before (top) and after the measurement (bottom).

Dropping the counterweight freely results in an acceleration, which is a function of the weight imbalance, as well as the polar moment of inertia of the moving parts. The vertical motion of the receivers $x(t)$ is governed by Eq. (15):

$$
J \ddot{\theta}=T_{M}-T_{S}-T_{\mathrm{F}} \quad x=Z \sin \theta
$$

where $J$ is the polar moment of inertia of the counterbalance device with respect to the pivot point; $\theta$ is the angle between the horizontal axis and the linkage arms; $T_{M}$ and $T_{S}$ are the moments caused by the counterweight $M$ and the receivers $S$, respectively, $T_{\mathrm{F}}$ is the torque due to friction and $Z$ is the length of the semi-arms holding the receivers. If the stroke of the receivers is small compared to the length of the parallel arms, i.e. the system only allows for small angular displacements, and the effect of the friction is negligible, Eq. (15) can be linearized as follows:

$$
\ddot{\theta}=\frac{A g}{J} \quad x=Z \theta
$$

where $g$ is the gravity acceleration, and $A$ is a constant proportional to the mass imbalance between the counterweight arm and the receiver arm. By taking the second derivative of $x(t)$ and substituting into it the expression of the angular 
1
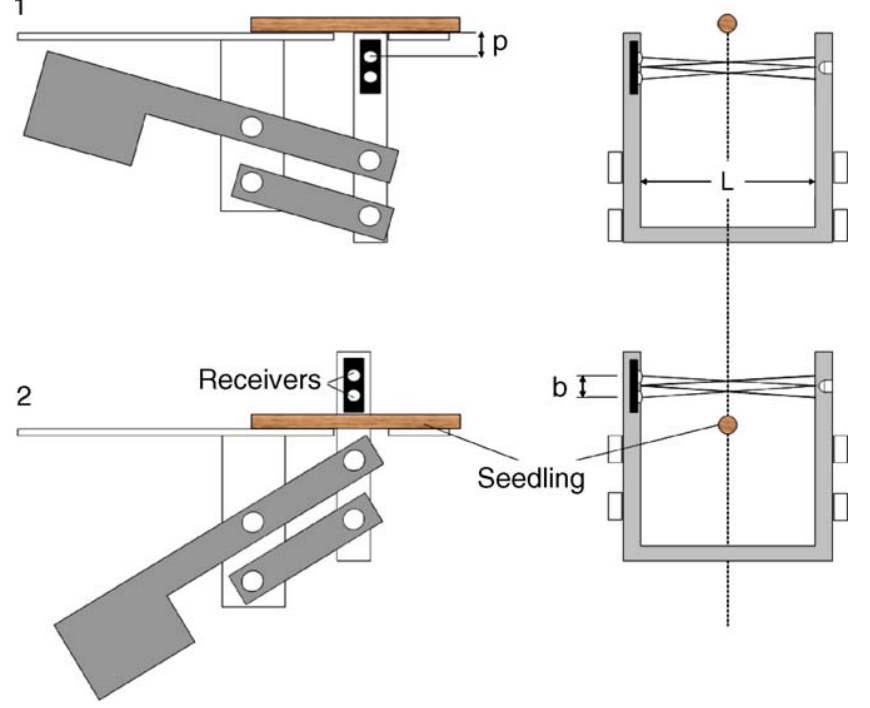

Fig. 5. Side-view and front-view of the counterbalanced diameter measurement device before (top) and after the measurement (bottom).

acceleration, we can write:

$$
a=\frac{Z A g}{J}
$$

which implies that the resulting acceleration of the receivers is constant.

The counterbalanced measurement device uses a widening beam, and thus, the measured diameter depends on the distance between the passage path and the receivers (see Fig. 2). When the operator places the object off-center errors are introduced. To compensate for this, a balance configuration was implemented, where two receiver sets (one transmitter combined with two opposing receivers) were placed in opposing orientation (see the front views on the right hand side of Fig. 5). Thus, when the object is placed off-center, the receivers closest to the object will measure a smaller diameter, which is compensated for by the opposite receiver set which measures a larger diameter. The resulting diameter was taken as the mean of the two simultaneous measurements. Further error containment was provided by projecting a laser line on the measurement surface to aid the operator in placing the seedling in the centerline of the measurement system.

Under constant receiver acceleration, the equation of motion becomes:

$$
x(t)=\frac{1}{2} a t^{2}+v_{0} t+x_{0}
$$

where $x_{0}$ and $v_{0}$ are the initial position and the initial velocity at an arbitrary initial time $t=0$. Instead of determining the receiver acceleration from a mechanical analysis, it will be shown that the acceleration can be directly computed from the timing signals $\Delta t_{\mathrm{p}}, \Delta t_{\mathrm{f}}$. This renders the diameter measurement independent of the factors that influence acceleration such as the mass imbalance, moment of inertia and, most importantly, friction in the pivot points. The distance between the initial position where the counterweight starts falling $\left(x_{0}=0\right)$ and the first receiver interruption $x\left(t_{1}\right)$ is termed $p$ (Fig. 5). This value is a constant defined by the system design, independent of the diameter of the object. Assuming an initial velocity $v_{0}=0$, inversion of Eq. (18) gives the time $t_{1}$ at which the first interruption event occurs, after traveling through $p$ :

$$
t_{1}=\sqrt{\frac{2 p}{a}}
$$

The velocity that the receivers have at $t_{1}$ is:

$$
v_{\mathrm{p}}=v\left(t_{1}\right)=a t_{1}=\sqrt{2 p a}
$$


To calculate the positions $x\left(t_{2}\right)$ and $x\left(t_{3}\right)$, Eq. (18) was applied with initial conditions at $t_{1}, x\left(t_{1}\right)=p$ and $v\left(t_{1}\right)=\sqrt{2 p a}$ yielding:

$$
x\left(t_{2}\right)=\frac{1}{2} a\left(t_{2}-t_{1}\right)^{2}+\sqrt{2 p a}\left(t_{2}-t_{1}\right)+p \quad x\left(t_{3}\right)=\frac{1}{2} a\left(t_{3}-t_{1}\right)^{2}+\sqrt{2 p a}\left(t_{3}-t_{1}\right)+p
$$

Earlier, Eq. (3) defined the flank time as $\Delta t_{\mathrm{f}}=t_{2}-t_{1}$, which allows for rewriting $x\left(t_{2}\right)$ from Eq. (21) as follows:

$$
x\left(t_{2}\right)-p=b=\frac{1}{2} a\left(\Delta t_{\mathrm{f}}\right)^{2}+\sqrt{2 p a} \Delta t_{\mathrm{f}}
$$

This equation contains the unknown acceleration $a$ as a function of the measured flank time $\Delta t_{\mathrm{f}}$ and the design constants $p$ and $b$. Similarly, recalling Eq. (4) which defined the pulse time $\Delta t_{\mathrm{p}}=t_{3}-t_{1}$, we can rewrite $x\left(t_{3}\right)$ from Eq. (21) as follows:

$$
x\left(t_{3}\right)-p=D=\frac{1}{2} a\left(\Delta t_{\mathrm{p}}\right)^{2}+\sqrt{2 p a} \Delta t_{\mathrm{p}}
$$

Solving Eq. (22) for the acceleration $a$ yields a single positive value as follows:

$$
a=\frac{4 p+2 b-4 \sqrt{p^{2}+p b}}{\left(\Delta t_{\mathrm{f}}\right)^{2}}
$$

which, substituted in Eq. (23) leads to the measurement equation for the diameter $D$ :

$$
D=(b-c)\left(\frac{\Delta t_{\mathrm{p}}}{\Delta t_{\mathrm{f}}}\right)^{2}+c \frac{\Delta t_{\mathrm{p}}}{\Delta t_{\mathrm{f}}}
$$

where the constant $c=2 \sqrt{p^{2}+p b}-2 p$ is solely related to the device design constants $p$ and $b$.

The counterbalanced configuration not only contains a constant acceleration, but also diverging light beams and consequently, the distance $b$ where the object passes the beams needs to be adjusted to $b_{\mathrm{H}}$, which is the distance between the light beams at the point of intersection according to Fig. 2:

$$
b_{\mathrm{H}}=b\left(\frac{L-T}{\sqrt{L^{2}+(b / 2)^{2}}}\right)
$$

The diameter measurement equation becomes finally:

$$
D=\left(b_{\mathrm{H}}-c\right)\left(\frac{\Delta t_{\mathrm{p}}}{\Delta t_{\mathrm{f}}}\right)^{2}+c \frac{\Delta t_{\mathrm{p}}}{\Delta t_{\mathrm{f}}}
$$

The assumption of constant acceleration from zero velocity implies that $\Delta t_{\mathrm{f}}$ is constant, since the receivers travel a constant distance through $p$. The acceleration itself only depends on the constants $b_{\mathrm{H}}, \Delta t_{\mathrm{f}}$ and $p$, and can be computed by substituting the constant $c=2 \sqrt{p^{2}+p b}-2 p$ into Eq. (24), leading to:

$$
a=\frac{2\left(b_{\mathrm{H}}-c\right)}{\left(\Delta t_{\mathrm{f}}\right)^{2}}
$$

Table 1 gives and overview of the considered configurations and the corresponding diameter measurement equations.

\section{Results}

Although the objective of this study deals with diameters of seedlings, the performance of the system cannot be assessed using seedlings because their diameter is not constant. Therefore, instead of seedlings, cylindrical objects were used for testing. The premise was that a sufficiently accurate device for cylindrical objects would suffice for seedlings as well. 
Table 1

Diameter measurement equations for five configurations

Configuration Diameter measurement equation

Parallel beam, constant velocity

Diverging beam, constant velocity

Diverging beam, constant velocity, sliding edge

Parallel beam, constant acceleration

Diverging beam, constant acceleration

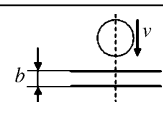

$D=b \frac{\Delta t_{\mathrm{p}}}{\Delta t_{\mathrm{f}}}$

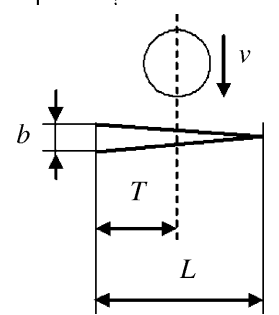

$D=b \frac{\Delta t_{\mathrm{p}}}{\Delta t_{\mathrm{f}}}\left(\frac{L-T}{\sqrt{L^{2}+(b / 2)^{2}}}\right)$

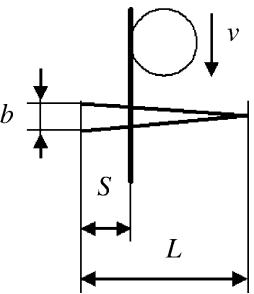

$D=\frac{L-S}{\left((1 / 2)+\left(\Delta t_{\mathrm{f}} / \Delta t_{\mathrm{p}}\right) \sqrt{(L / b)^{2}+(1 / 4)}\right)}$

$D=(b-c)\left(\frac{\Delta t_{\mathrm{p}}}{\Delta t_{\mathrm{f}}}\right)^{2}+c \frac{\Delta t_{\mathrm{p}}}{\Delta t_{\mathrm{f}}}$

$c=2 \sqrt{p^{2}+p b}-2 p, \quad a=\frac{2(b-c)}{\left(\Delta t_{\mathrm{f}}\right)^{2}}$

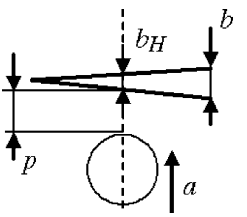

$D=\left(b_{\mathrm{H}}-c\right)\left(\frac{\Delta t_{\mathrm{p}}}{\Delta t_{\mathrm{f}}}\right)^{2}+c \frac{\Delta t_{\mathrm{p}}}{\Delta t_{\mathrm{f}}} \quad b_{\mathrm{H}}=b\left(\frac{L-T}{\sqrt{L^{2}+(b / 2)^{2}}}\right)$,

$c=2 \sqrt{p^{2}+p b}-2 p, \quad a=\frac{2\left(b_{\mathrm{H}}-c\right)}{\left(\Delta t_{\mathrm{f}}\right)^{2}}$

\subsection{Sliding-edge arrangement with constant velocity}

Cylindrical drill bit blanks were used to assess the performance of the sliding-edge device. The reference diameters were measured with a slide micrometer with an accuracy of $0.01 \mathrm{~mm}$ and the diameter measurement equation used was Eq. (14). The model constants $L, S$, and $b$ were measured using a slide micrometer, but one assumption was that the infrared radiation source size was infinitely small, which is inaccurate. This model error plays a more important role when the distance between the object trajectory and the receivers is small such as in the sliding-edge case. In addition, the exact switching point of the optical receivers was assumed in the center, another assumption that may be inaccurate.

In Table 2, a comparison among the measured and reference diameter values is shown. The maximum error (column 6) was approximately $9.5 \%$. The variability among the measurements (column 4) was small, considering the objects were slid past the receivers by a human operator. The error standard deviation shows a positive correlation with the diameter, which is logical since the measurement time increases for larger diameters. 
Table 2

Comparison of true and measured diameters using sliding-edge device (12 repetitions per diameter)

\begin{tabular}{rllllr}
\hline & True diameter $(\mathrm{mm})$ & Mean measured diameter $(\mathrm{mm})$ & S.D. $(\mathrm{mm})$ & Coefficient of variation $(\%)$ & Error $(\%)$ \\
\hline 1 & 3.17 & 2.87 & 0.01 & 0.45 & 9.49 \\
2 & 3.57 & 3.31 & 0.02 & 0.68 & 7.35 \\
3 & 3.97 & 3.77 & 0.02 & 0.45 & 5.04 \\
4 & 4.34 & 4.19 & 0.02 & 0.59 & 3.40 \\
5 & 4.74 & 4.62 & 0.02 & 0.34 & 2.61 \\
6 & 5.12 & 5.05 & 0.03 & 0.66 & 1.33 \\
7 & 5.53 & 5.47 & 0.05 & 0.98 & -0.30 \\
8 & 5.92 & 5.94 & 0.05 & 0.84 & 0.04 \\
9 & 6.33 & 6.33 & 0.04 & 0.66 & -0.63 \\
10 & 6.69 & 6.73 & 0.06 & 0.83 & -0.28 \\
11 & 7.91 & 7.93 & 0.10 & 1.27 & -2.08 \\
12 & 9.46 & 9.66 & 0.08 & 0.8 & \\
\hline
\end{tabular}

Table 3

Comparison of true and measured diameters using counterbalanced device ( 24 repetitions per diameter)

\begin{tabular}{rlllrr}
\hline & True diameter $(\mathrm{mm})$ & Mean measured diameter $(\mathrm{mm})$ & S.D. $(\mathrm{mm})$ & \multicolumn{2}{c}{ Coefficient of variation $(\%)$} \\
\hline 1 & 3.94 & 3.95 & 0.01 & 0.37 & -0.24 \\
2 & 4.35 & 4.35 & 0.02 & 0.56 & 0.05 \\
3 & 4.74 & 4.77 & 0.03 & 0.54 & 0.95 \\
4 & 5.13 & 5.18 & 0.03 & 0.49 & -0.12 \\
5 & 5.55 & 5.54 & 0.02 & 0.45 & -0.05 \\
6 & 6.35 & 6.35 & 0.03 & 0.51 & 0.09 \\
7 & 6.72 & 6.73 & 0.03 & 0.52 & -0.08 \\
8 & 7.88 & 7.87 & 0.04 & 0.51 & 1.02 \\
9 & 9.46 & 9.56 & 0.11 & 1.18 & \\
\hline
\end{tabular}

\subsection{Counterbalanced configuration with constant acceleration}

The counterbalanced device was evaluated using standard drill bits which were measured as reference diameters with slide micrometer with an accuracy of $0.01 \mathrm{~mm}$. In Table 3, the results are shown.

The equations used for the diameter measurement were Eqs. (26) and (27), and the model parameters $L, T, b$, and $p$ were measured using a slide micrometer as: $L=44 \mathrm{~mm}, T=22 \mathrm{~mm}, b=2.7 \mathrm{~mm}, p=3.8 \mathrm{~mm}$. The error variability (column 4) is proportional to the mean diameter, as expected, since the measurement time increases with the diameter. The resulting measurement error is much smaller than in the sliding-edge case which shows that the counterbalanced method is superior in performance, owing to a properly validated and correct model.

\section{Conclusions}

This paper describes the development of optical time-of-flight based methods for the measurement of diameters of cylindrical objects. The research was inspired by the need for measuring the root collar diameter (RCD) of pine seedlings, but applies to any case where a real time diameter measurement of opaque cylindrical objects is desired. Four arrangements were analysed: (1) a parallel light beam arrangement with constant object velocity, (2) a diverging light beam arrangement with constant object velocity, (3) a diverging light beam arrangement with constant object velocity and a sliding edge for guidance and (4) a counterbalanced arrangement with a diverging beam and constant receiver acceleration. Methods 3 and 4 were considered best candidates and implemented and evaluated.

Method 3 relied on the operator sliding the object along a guidance edge. After validating the model that describes the interaction between a sliding object and the receivers, a systematic error was found with a maximum of $9.5 \%$. This discrepancy was most likely owing to non-valid model assumptions. In the counterbalanced configuration (method 4) the receivers were moved with respect to a stationary object. Here the operator can simply place the object on a measurement table (aided by a projected laser line) and obtain a diameter measurement at the push of a button. This 
method was found superior in reproducibility and accuracy as well as ease of operation compared to the sliding-edge configuration. The maximum systematic error was $0.1 \mathrm{~mm}$, and the variability among 24 repetitions ranged from $0.02 \mathrm{~mm}$ for the smallest diameter $(3.94 \mathrm{~mm})$ and increased to $0.11 \mathrm{~mm}$ for the largest diameter $(9.46 \mathrm{~mm})$.

The sliding-edge device is very simple in design, requiring only a transmitter, dual receivers and a guide, but its accuracy was not sufficient. This was mainly because of erroneous model assumptions, not because of the inability of the operator to maintain a constant velocity. The counterbalanced device is more complicated in design; it requires dual transmitters, four receivers, laser line guidance, and a mechanism to drop the counterweight. On the other hand, the dimensions and the mass of the counterweight can be chosen arbitrarily, since the measurements are obtained from the timing information in which even mechanical friction is subsumed. Since the total cost of the measurement systems includes computer hardware, a counter/timer board and software, the cost of the measurement device hardware plays a relatively small role. Since the counterbalanced device is more accurate, reliable and user friendly than the sliding-edge device, it has the optimal cost/performance ratio.

In the future, the proposed methods could be used to develop a seedling counter on a harvester (lifter). Since the plants are transported on a conveyor belt with relatively constant velocity and orientation, a dual receiver arrangement with balance configuration, similar to the counterbalanced device, could be applied. This would not only allow for an accurate seedling count (which is currently unreliable since it is based on a reference volume) but also provide the diameter distribution which could be used as a batch quality indicator.

\section{References}

Byrne, J., Singh, S., 1998. Precise image segmentation for forest inventory. Internal report CMU-RI-TR-98-14. Carnegie Mellon University, Pittsburgh, PA. Available at: http://www.ri.cmu.edu/pub_files/pub1/byrne_jeffrey_1998_1/byrne_jeffrey_1998_1.pdf (accessed December 29, 2005).

Clark, N.A., Wynne, R.H., Schmoldt, D.L., Winn, M., 2000. An assessment of the utility of a non-metric digital camera for measuring standing trees. Comput. Electron. Agric. 28, 151-169.

Delwiche, M., Voorhees, J., 2003. Optoelectronic system for counting and sizing of field-grown deciduous trees. Trans. ASAE 46 (3), $877-882$.

Gasvoda, D., Herzberg, D., 1992. MTDC seedling counter field tests. Pub. 9224-2829 MTDC, USDA Forest Service, Missoula Montana.

Grift, T.E., Hofstee, J.W., 1997. Measurement of velocity and diameter of individual fertiliser particles by an optical method. J. Agric. Eng. Res. 66 (3), 235-238.

Kranzler, G.A., 1988. An opto-electronic tree seedling counter. ASAE paper No. 88-7521, ASAE. St. Joseph, MI.

Kranzler, G.A., Rigney, M.P., 1989. Feasibility of machine vision for tree seedling grading and root growth measurement. Pub. 9024-2814-MTDC, USDA Forest Service, Missoula Montana.

Maw, B.W., Brewer, H.L., Thompson, S.J., 1986. Photoelectric transducer for detecting seedlings. Trans. ASAE 29 (4), $912-916$.

McDonald, T.P., Rummer, R.B., Grift, T.E., 2003. Diameter sensors for tree-length harvesting systems. In: Wide, M.I., Baryd, B. (Eds.), Proceedings of the Second Forest Engineering Conference. Vaxjo, Sweden. Skogforsk, Uppsala Science Park, Uppsala, Sweden, pp. 45-54.

Moulton, R.J., 1999. Tree planting in the United States 1997. Tree Planters' Notes 49(1), 5-15. USDA Forest Service. Available at: http://www.rngr.net/ (accessed December 29, 2005).

Rigney, M.P., Kranzler, G.A., 1988. Machine vision for grading southern pine seedlings. Trans. ASAE 31 (2), $642-646$.

South, D. 2005. Tree Planters' Notes 51(1), 2-3. USDA Forest Service. Available at: http://www.rngr.net/ (accessed December 29, 2005).

USDA Forest Service, 2002. State and Private Forestry, Cooperative Forestry, Washington Office, unpublished data.

Wilhoit, J.H., Kutz, L.J., Fly, D.E., South, D.B., 1994. PC-based multiple camera machine vision systems for pine seedling measurement. Appl. Eng. Agric. 10 (6), 841-847. 